\title{
Нейтрофильные внеклеточные
}

\section{ловушкИ: значение для \\ диагностики и прогноза COVID-19}

\author{
Кассина Д.В. • Василенко И.А. ${ }^{1,2}$ • Гурьев А.С., ${ }^{1,3}$ • Волков А.Ю. ${ }^{3}$ • Метелин В.Б.' ${ }^{1}$
}

Кассина Дарья Валерьевна - науч. сотр. научно-исследовательской лаборатории'; ORCID: https://orcid. org/0000-0002-6759-9121

Василенко Ирина Анатольевна д-р мед. наук, профессор, вед. науч. сотр. научно-исследовательской лаборатории'; профессор кафедры прикладной математики и программирования²; ORCID: http:// orcid.org/0000-0002-6374-9786 $\triangle 115035$, г. Москва, ул. Садовническая, 33/1, Российская Федерация. Тел.: +7 (495) 9515497. E-mail: vasilenko0604@gmail.com

Гурьев Александр Сергеевич - PhD, ст. науч. сотр. научно-исследовательской лаборатории'; науч. сотр. ${ }^{3}$; ORCID: https://orcid.org/0000-0001-8823-7819

Волков Алексей Юрьевич - канд. физ.-мат. наук, генеральный директор ${ }^{3}$; ORCID: https://orcid.org/0000-0001-5110$553 \mathrm{X}$

Метелин Владислав Борисович канд. биол. наук, вед. науч. сотр. научноисследовательской лаборатории'; доцент кафедры философии, манусологии и теологии; ORCID: http:// orcid.org/0000-0003-0600-5729
Актуальность. В патогенезе COVID-19 важным элементом противовирусной защиты выступает врожденный клеточный иммунитет, включающий в том числе полиморфноядерные нейтрофилы, склонные к нетозной трансформации. При этом нейтрофилы не только служат маркером острой инфекции, но и, будучи источником нейтрофильных внеклеточных ловушек (НВЛ), играют ключевую роль в развитии тромботических осложнений, приводящих к острой дыхательной недостаточности при COVID-19. Цель - установить диагностическое и прогностическое значение уровня внеклеточных нейтрофильных ловушек у пациентов c COVID-19. Материал и методы. Проведен мониторинг уровня НВЛ периферической крови у 34 пациентов с COVID-19 (средний возраст $67 \pm 15,8$ года), госпитализированных в ГБУЗ МО МОНИКИ им. М.Ф. Владимирского. Контрольную группу составили 54 практически здоровых добровольца (средний возраст $52 \pm 11,5$ года). Из 2 мкл цельной крови готовили мазки по типу «монослой», окрашивали по Романовскому - Гимзе и с помощью системы автоматического микроскопа МЕКОС-Ц2 (ООО «Медицинские компьютерные системы (МЕКОС)») подсчитывали массив не менее 200 клеточных структур, включающий нативные неразрушенные и трансформированные нейтрофилы. Результаты. У пациентов c COVID-19 зарегистрирован высокий уровень НВЛ - 14,5\% (2,9-28,6\%) против 5,0\% (1,8-11,9\%) в контрольной группе ( $p<0,0001)$. 23,5\% больных, получавших простую респираторную поддержку, продемонстрировали уровень НВЛ $12 \%$ (8,1-22,3\%), тогда как у 17,6\% пациентов, которые были подключены к искусственной вентиляции легких, уровень НВЛ оказался в 1,5 раза выше - 17,9\% (12,3-28,2\%) ( $p<0,05)$. В 11,8\% случаев с летальным исходом уровень НВЛ достигал 19\% (16,5-26\%) (p<0,05). Мониторингуровня НВЛ в крови 9 больных от момента поступления до момента перевода в отделение реанимации и интенсивной терапии/выписки или смерти показал, что снижение уровня обнаруженных НВЛ отражает улучшение клинического состояния и эффективность проводимой терапии. Рост уровня НВЛ, напротив, может свидетельствовать об ухудшении и риске негативного развития событий. Заключение. Выявлены некоторые патофизиологические механизмы развития COVID-19, связанные с компартментом нейтрофилов. Установлено, что для больных с коронавирусной инфекцией характерно наличие высокого уровня НВЛ, который в 3 раза и более превышает показатели практически здоровых добровольцев и свидетельствует о сбое иммунных механизмов защиты и развитии неадекватного воспалительного ответа. Повышение нейтрофильных внеклеточных ловушек в мазках цельной крови более 16\% может служить критерием негативного прогноза течения заболевания и риска летального исхода.

Ключевые слова: COVID-19, нейтрофильные внеклеточные ловушки, нетоз

Для цитирования: Кассина ДВ, Василенко ИА, Гурьев АС, Волков АЮ, Метелин ВБ. Нейтрофильные внеклеточные ловушки: значение для диагностики и прогноза COVID-19. Альманах клинической медицины. 2020;48(S1):S43-50. doi: 10.18786/20720505-2020-48-029.

Поступила 13.07.2020; доработана 28.07.2020; принята к публикации 29.07.2020; опубликована онлайн 11.08.2020

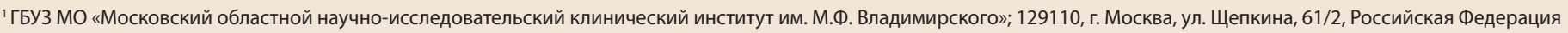

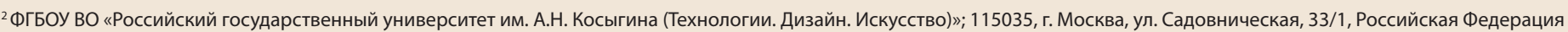
${ }^{3}$ ООО «Медтехнопарк»; 117292, г. Москва, ул. Профсоюзная, 8-2-383, Российская Федерация 
B ирусная инфекция (COVID-19), возбудителем которой является бета-коронавирус SARS-CoV-2, в течение нескольких месяцев охватила подавляющую часть стран и континентов. Новая пандемия оказалась серьезным вызовом для общественного здравоохранения вследствие недостаточной изученности вируса и постоянно меняющейся информации о механизмах передачи, клинических проявлениях, осложнениях, диагностике, эффективных профилактических и терапевтических мероприятиях. По мнению специалистов, патофизиология заболевания непосредственно связана с неконтролируемым прогрессированием системного воспаления, завершающегося распространенным иммунотромбозом микроциркуляторного русла и развитием на этом фоне жизнеугрожающих осложнений в виде острого респираторного дистресс-синдрома (ОРДС) и полиорганной недостаточности [1-3].

Всплеск клинических проявлений заболевания с выраженным системным течением ассоциируют с неупорядоченным высвобождением цитокинов (так называемым цитокиновым штормом), включая про- и противовоспалительные цитокины, хемокины, а также проапоптотические медиаторы (IL-1, IL-2, IL-6, IL-7, IFN- $\gamma$, TNF- $\alpha$, IP-10, MCP-1, G-CSF MIP-1A и др.) $[4,5]$.

Важное значение в патогенезе COVID-19 имеют клеточные компоненты иммунной системы. Особое внимание привлекают такие факторы врожденного иммунитета, как полиморфноядерные нейтрофилы (PMNs) и нетоз (NETosis), приводящий к образованию нейтрофильных внеклеточных ДНК-ловушек (НВЛ) с ярко выраженными иммуногенными, тромбогенными и токсическими свойствами. При этом общий уровень нейтрофилов служит маркером острого инфекционного процесса, а нетозно трансформированные нейтрофилы, будучи источником НВЛ, играют ключевую роль в развитии тромботических осложнений, приводящих к острой дыхательной недостаточности при COVID-19 $[6,7]$. Так, вскрытие пациентов, погибших от COVID-19, выявило выраженную инфильтрацию нейтрофилов в легочных капиллярах, их выход в альвеолярное пространство, острое воспаление капилляров с отложением фибрина, а также нейтрофильный мукозит [8]. Новые данные о НВЛ могут привести к пересмотру патофизиологической картины тромбозов и заболеваний дыхательных путей при вирусных инфекциях.

Общепринятых стандартных методов диагностики и исследования НВЛ не разработано.
Предлагаемые методики в основном сложные, дорогостоящие и времязатратные [9]. Они хорошо подходят для фундаментальных медико-биологических исследований, но малоприменимы в практической лабораторной диагностике. Нами разработан максимально простой, общедоступный метод оценки уровня этотически трансформированных фагоцитов, который может использоваться в рутинной лабораторной практике и быть полезным для принятия быстрых решений в экстренной медицине [10]. Определение уровня НВЛ позволяет контролировать дисрегуляционные изменения в иммунной системе на более ранних этапах развития патологического процесса.

Цель настоящей работы - установить диагностическое и прогностическое значение уровня внеклеточных нейтрофильных ловушек у пациентов с COVID-19.

\section{Материал и методы}

В проспективное когортное исследование включены 34 пациента с COVID-19 (15 женщин, 19 мужчин), госпитализированных в ГБУЗ МО МОНИКИ им. М.Ф. Владимирского. Средний возраст обследованных больных составил $67 \pm 15,8$ года (минимум - 37 лет, максимум 95 лет). Большинство (92\%) пациентов имели в анамнезе сопутствующие заболевания, в том числе $53 \%$ страдали гипертонической болезнью I-III степени, $20 \%$ сахарным диабетом 2-го типа. $20(58,8 \%)$ пациентов находились во временном инфекционном отделении, $14(41,2 \%)$ - в палате интенсивной терапии, из них $6(17,6 \%)$ были подключены к искусственной вентиляции легких (ИВЛ), 4 (11,8\%) пациента скончались.

Контрольную группу составили 54 практически здоровых добровольца (30 женщин, 24 мужчины) в возрасте от 28 до 72 лет (средний возраст $52 \pm 11,5$ года).

Протокол исследования был одобрен локальным этическим комитетом при ГБУЗ МО МОНИКИ им. М.Ф. Владимирского (заседание № 5 от 13 мая 2020 г.). Критерии включения в исследование: предварительный диагноз COVID-19, согласие пациента или его представителя на участие в исследовании. Критерии исключения: отказ пациента или его представителя от участия в исследовании, не подтвержденный диагноз COVID-19.

Исследование проводили в динамике с трехдневным интервалом. Периферическую кровь заготавливали в вакуумные пробирки с ЭДТА и из 2 мкл цельной крови готовили мазки по 


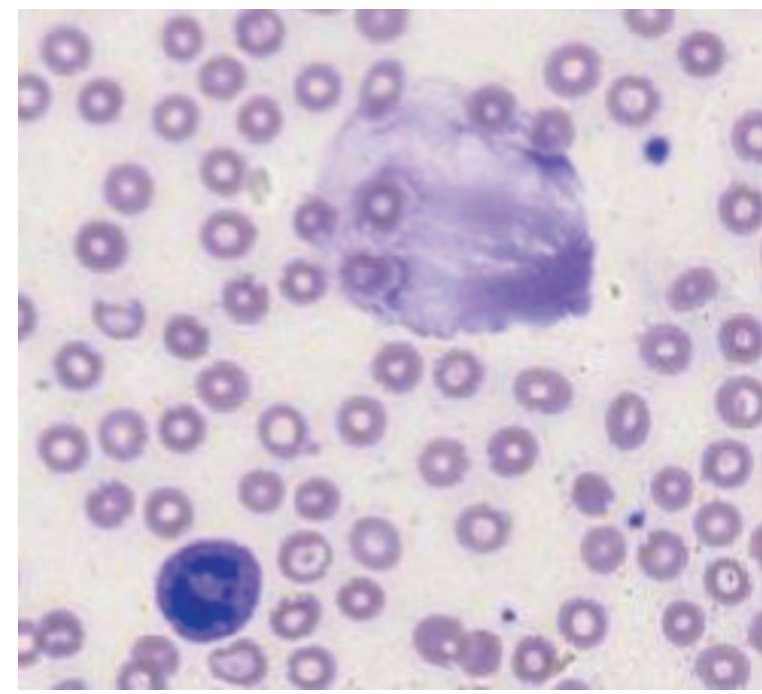

Рис. 1. Микрофотография: сегментоядерный нейтрофил (снизу) и нейтрофильная внеклеточная ловушка (сверху), визуализируемые в мазке крови типа «монослой», окрашенном по Романовскому - Гимзе (×500) (фото из личного архива авторов, клинический материал)

типу «монослой» [11]. Далее мазки окрашивали по Романовскому - Гимзе и подсчитывали 200 клеточных структур, включая нативные неразрушенные нейтрофилы $\left(\mathrm{N}_{\text {нат }}\right)$ и НВЛ $\left(\mathrm{N}_{\text {нвл }}\right)$, с помощью системы автоматической микроскопии МЕКОС-Ц2 (ООО «Медицинские компьютерные системы (МЕКОС)», Россия).

Уровень НВЛ (\%НВЛ) в мазке, отражающий долю нетотически трансформированных нейтрофилов, циркулирующих в периферической крови [10], рассчитывали по формуле

$$
\% \text { НВЛ }=N_{\text {нвл }} /\left(N_{\text {нат }}+N_{\text {нвл }}\right) \text {, }
$$

где $N_{\text {нвл }}$ - количество нейтрофильных внеклеточных ловушек, $N_{\text {нат }}$ - количество нативных нейтрофилов.

Показатель маркера, усредненный по группе, дан как медианное значение (минимальное - максимальное значение). Распределение полученных показателей представлено в виде диаграммы размаха, на которой границами боксплота служат 25- и 75-й процентили, линия в середине отображает медианное значение, крестом отмечено среднее значение, концы «усов»- минимальное и максимальное значения. Статистические различия между выборками оценивали при помощи U-критерия Манна - Уитни. Корреляцию между \%НВЛ и маркерами воспаления определяли при помощи теста ранговой корреляции Спирмена. Для всех видов анализа статистически значимыми считали различия при $\mathrm{p}<0,05$. Расчеты проводили в программном пакете Statistica 12 (TIBCO Software Inc., США).

\section{Результаты}

НВЛ хорошо визуализируются в мазках цельной крови и представляют собой структуры, занимающие объем, в 10-15 раз превышающий объем исходных клеток, и состоящие из нитей деконденсированного хроматина, на поверхности которых иммобилизованы многочисленные антимикробные пептиды и ферменты гранулоцитов (рис. 1).

В нашем исследовании уровень НВЛ практически здоровых доноров составил 5,0\% (1,8$11,9 \%)$, тогда как у пациентов с COVID-19 \%НВЛ был статистически значимо $(\mathrm{p}<0,0001)$ увеличен - 14,5\% (2,9-28,6\%) (рис. 2).

При сравнении уровней НВЛ и традиционных маркеров воспаления (С-реактивный белок, количество нейтрофилов и лейкоцитов) не было установлено четкой корреляции между этими показателями (рис. 3). Напротив, обращала на себя внимание незначительная нейтропения у обследованных больных, по-видимому, имеющая компенсаторный характер.

Мы не выявили значимых изменений уровня НВЛ в крови у пациентов с различной степенью поражения легких по данным компьютерной томографии (КТ). У всех больных с легкой степенью тяжести КТ-1 (вовлечение паренхимы легкого $\leq 25 \%)$, среднетяжелой КТ-2 (вовлечение паренхимы легкого 25-50\%), тяжелой КТ-3 (вовлечение паренхимы легкого 50-75\%)

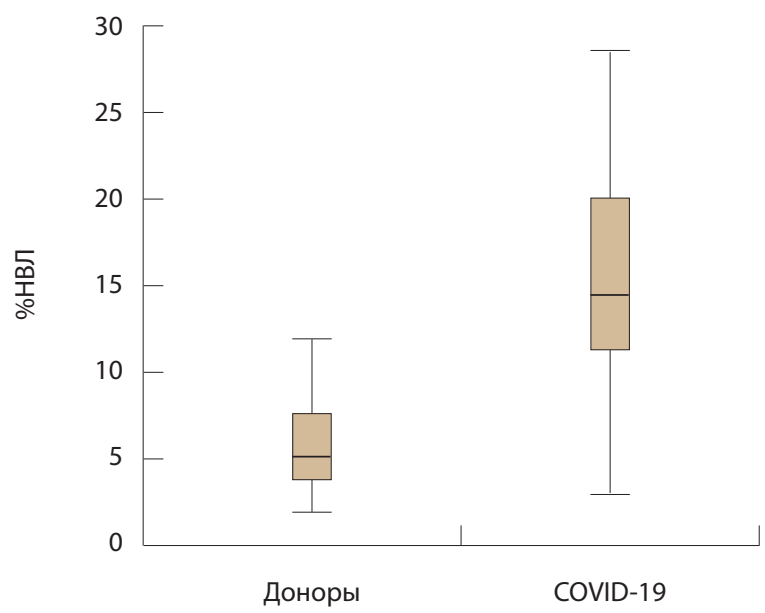

Рис. 2. Уровень нейтрофильных внеклеточных ловушек (НВЛ) у практически здоровых доноров и пациентов с COVID-19; $\mathrm{p}<0,0001$ 

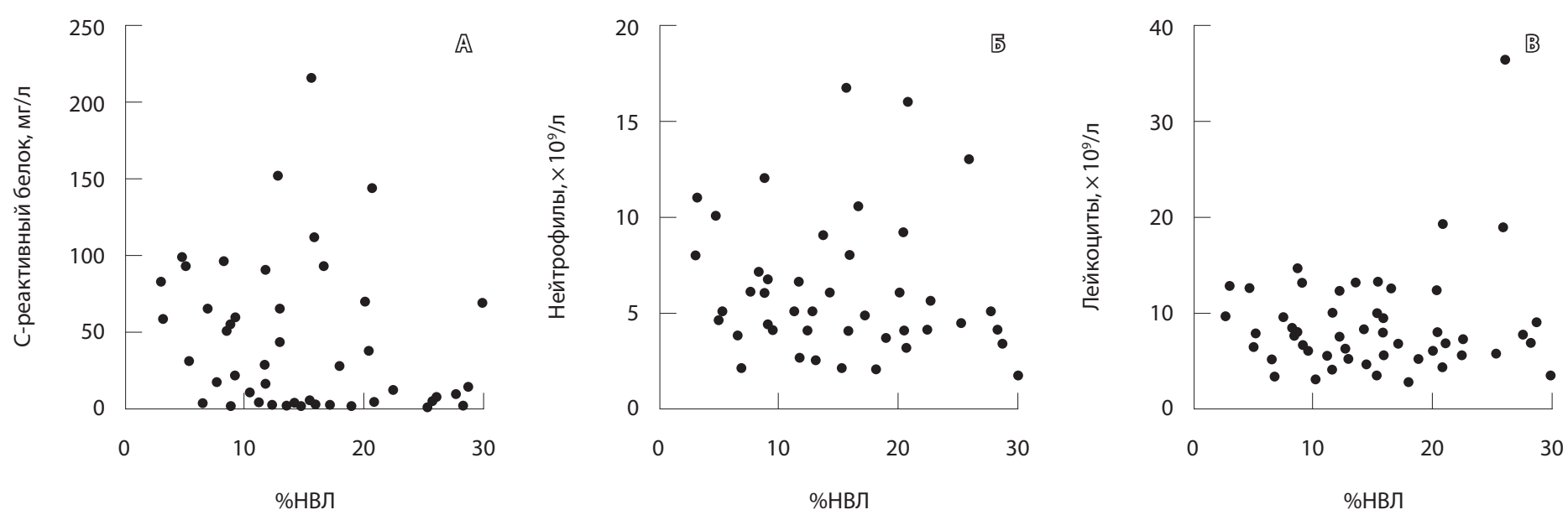

Рис. 3. Связь между уровнями нейтрофильных внеклеточных ловушек (НВЛ) и маркерами воспаления: С-реактивным белком (А), количеством нейтрофилов (Б), количеством лейкоцитов (В)

и критической КТ-4 (вовлечение паренхимы легкого $\geq 75 \%)$ \%НВЛ сохранялся на высоких значениях: $18,0 \%(4,5-28,6 \%), 16,2 \%(5,0-21,0 \%)$, $13,7 \%(2,9-28,2 \%)$ и $13,8 \%(8,1-26,0 \%)$ соответственно. Не исключено, что такой результат связан с небольшой выборкой обследованных пациентов.

Важные результаты были получены при анализе образцов цельной крови 14 пациентов c COVID-19, которые в связи с усилением симптомов дыхательной недостаточности были по показаниям переведены в отделение реанимации и интенсивной терапии (ОРИТ) для проведения респираторной терапии (рис. 4).

У 8 (23,5\%) пациентов, получавших простую респираторную поддержку, уровень НВЛ

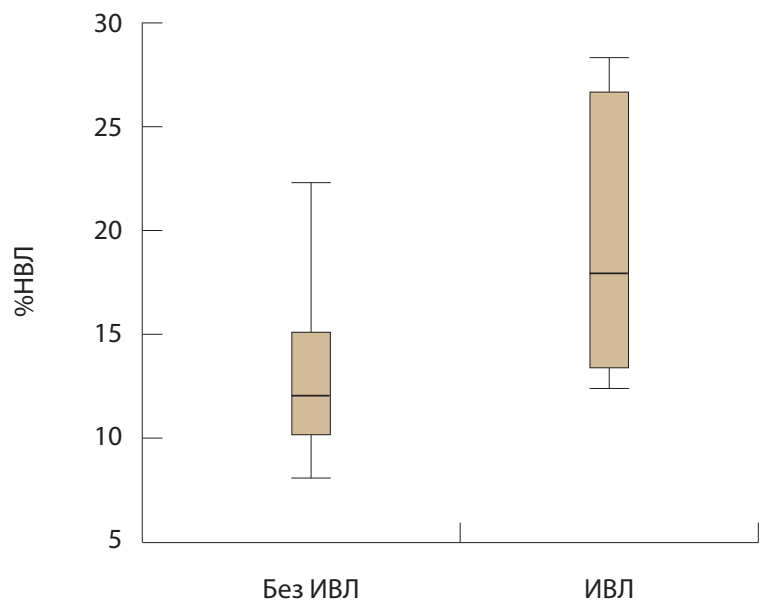

Рис. 4. Уровень нейтрофильных внеклеточных ловушек (НВЛ) у пациентов с острой дыхательной недостаточностью, нуждающихся в проведении респираторной терапии или искусственной вентиляции легких (ИВЛ); $p<0,05$ составил $12,0 \%(8,1-22,3 \%)$. А у $6(17,6 \%)$ пациентов, которые были подключены к ИВЛ, \%НВЛ оказался в 1,5 раза выше - 17,9\% (12,3-28,2\%) $(\mathrm{p}<0,05)$.

Мы сравнили уровень НВЛ среди умерших и выживших пациентов с COVID-19 (рис. 5). У 30 выживших больных \%НВЛ составил в среднем 13,3\%, тогда как у 4 пациентов с летальным исходом - 19,1\% (16,5-26\%, p<0,05). Можно предположить, что выявление в циркуляции высокого (более $16 \%$ ) уровня нетозно трансформированных нейтрофилов может служить критерием негативного прогноза исхода заболевания.

У большинства больных кровь была исследована однократно, однако у 9 из 34 пациентов было проанализировано 3 и более образцов

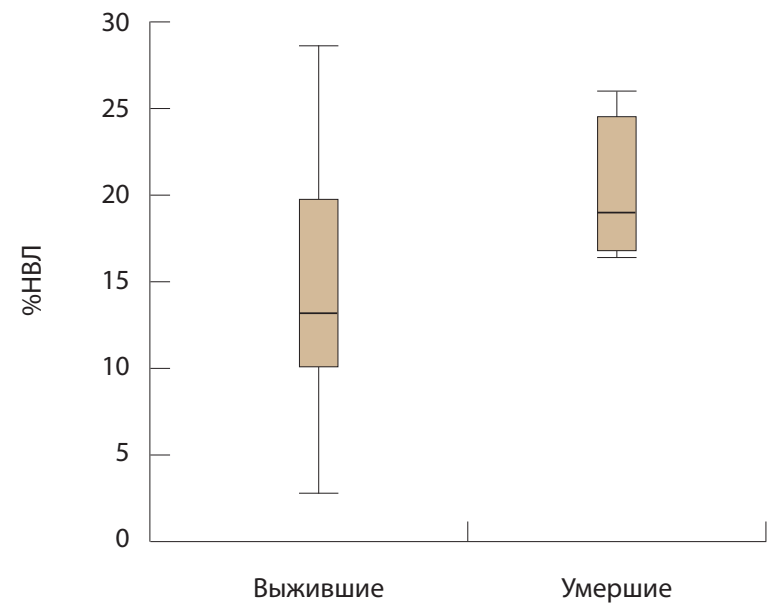

Рис. 5. Уровень нейтрофильных внеклеточных ловушек (НВЛ) у выживших и умерших пациентов с COVID-19; p <0,05 


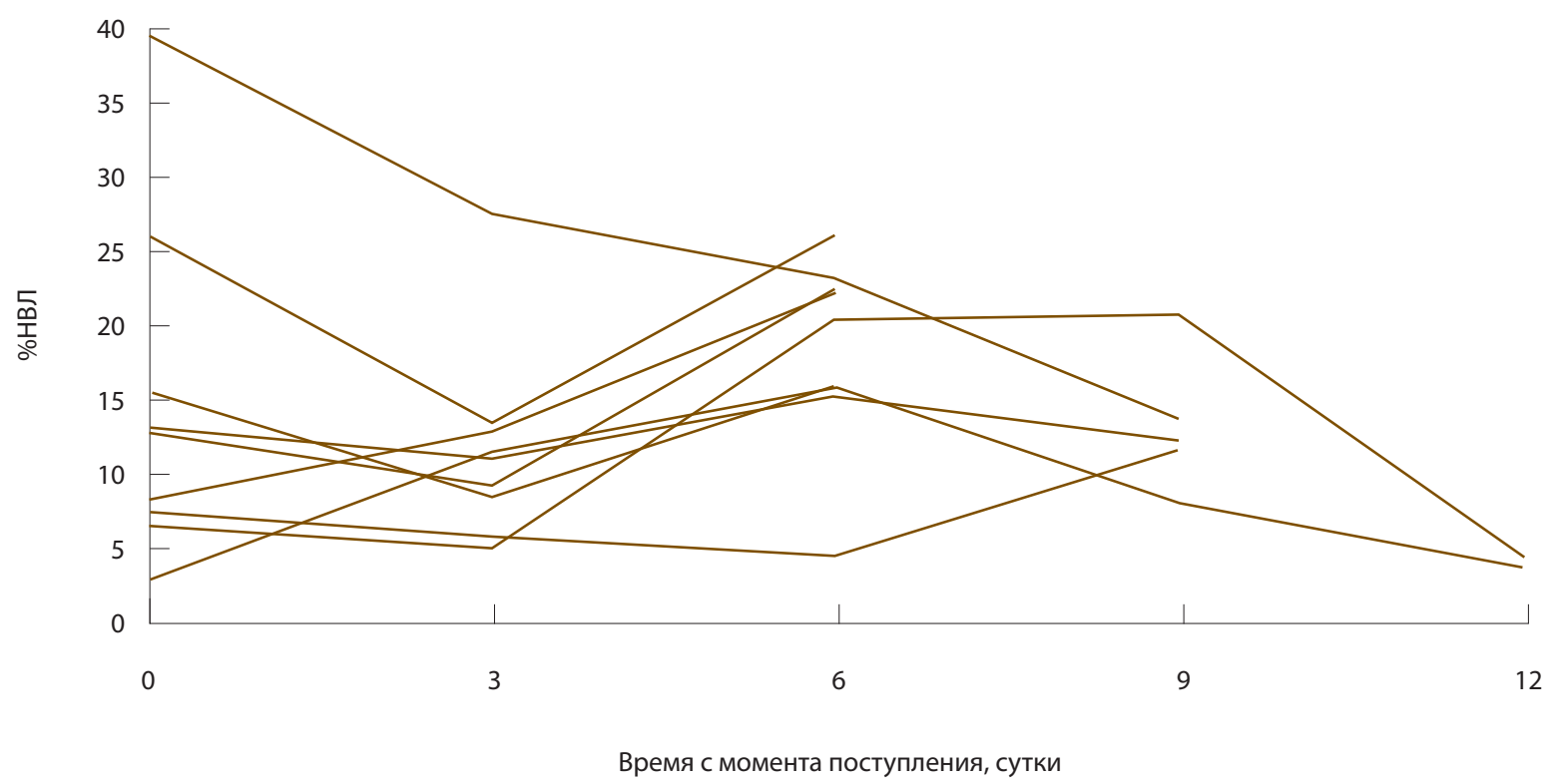

Рис. 6. Уровень нейтрофильных внеклеточных ловушек (НBЛ) у 9 пациентов с COVID-19, обследованных в динамике

крови в динамике от момента поступления до момента перевода в ОРИТ / выписки или смерти (рис. 6).

Снижение исходно высокого уровня НВЛ, обнаруженных в крови пациентов, отражало улучшение клинического состояния и эффективность проводимой патогенетической и симптоматической терапии. И напротив, рост \%НВЛ был связан с ухудшением общего состояния и риском негативного развития событий: перевод в реанимационное отделение с последующей респираторной терапией или летальный исход.

\section{Обсуждение}

Исследования последних лет изменили мнение о достаточно пассивной роли нейтрофилов в эффекторных реакциях иммунной системы. Новые данные о гетерогенности, высокой пластичности и широкой функциональности позволили выявить иные, неизвестные ранее антибактериальные стратегии этих клеток. Одна из них нетоз - механизм гибели нейтрофилов, индуцированный определенными праймерами и сопровождающийся формированием НВЛ [12-14].

Процесс нетоза представляет собой генетически детерминированную запрограммированную гибель нейтрофилов и связан с последовательными необратимыми морфологическими изменениями этих клеток: деконденсацией хроматина, фрагментацией и дезагрегацией ядерных мембран, смешиванием ядерных структур с цитоплазмой и высвобождением их вместе с содержимым гранул во внеклеточное пространство при нарушении целостности цитоплазматической мембраны [15].

Образование НВЛ направлено на реализацию реакций, осуществляемых клеточными элементами неспецифической иммунной защиты организма. Однако, в отличие от фагоцитоза, неконтролируемый нетоз приводит к избыточному появлению НВЛ, токсичных для организма, способствует прогрессированию воспалительных и аутоиммунных процессов, провоцирует развитие тромбозов и может быть причастным к патогенезу сердечно-сосудистых и онкологических заболеваний $[14,15]$.

Зарегистрированные нами высокие значения НВЛ в мазках цельной крови у обследованных пациентов полностью согласуются с данными других авторов, полученными при анализе молекулярных маркеров нетоза [3, 7-9]. Представляется очевидной роль НВЛ в негативной регуляции прогрессирующего системного воспаления при COVID-19, что привлекает внимание исследователей к детальному изучению нарушений в компартменте лейкоцитов.

При анализе динамики «классических» критериев острого воспаления, таких как количество лейкоцитов, нейтрофилов, С-реактивный белок, было отмечено, что лейкоцитоз и нейтрофилия при коронавирусной инфекции имеют отрицательное прогностическое значение и могут 
свидетельствовать о тяжелом течении респираторного процесса и его исходе $[8,16,17]$. Кроме того, по мнению исследователей, повышенный уровень нейтрофилов служит источником избыточных НВЛ $[8,17]$. Вместе с тем при сопоставлении результатов, полученных различными научными группами, следует учитывать возможность несоответствия сроков лабораторного исследования длительности течения и тяжести коронавирусной инфекции. Так, на определенных этапах заболевания наряду с нейтрофилией могут выявляться случаи нейтропении вследствие высокой инфильтрации тканей нейтрофилами и истощения миелопоэза. Имеющиеся в настоящее время сведения остаются достаточно противоречивыми и имеют определенные ограничения, которые связаны с гетерогенностью в выборе пациентов, различным дизайном исследований, высоким риском систематической ошибки.

С учетом уже известных особенностей патогенеза коронавирусной инфекции чрезмерное рекрутирование и активацию нейтрофилов с последующей массивной генерацией НВЛ можно рассматривать в качестве одного из основных факторов, влияющих на острое повреждение ткани легких. При реакции высвобождения биологически активных и агрессивных клеточных факторов (лейкотриенов, протеаз, оксидантов, фактора агрегации тромбоцитов и др.) повышается проницаемость сосудов, происходит отложение фибрина в альвеолах, микротромбообразование, что способствует нарушению процессов вентиляции легких с накоплением в альвеолах избыточной жидкости $[8,16]$. Эти события провоцируют развитие ОРДС, коагулопатию, полиорганную недостаточность и аутоиммунные заболевания $[18,19]$.

По данным ряда авторов, среди пациентов c COVID-19, страдающих ОРДС и нуждающихся в проведении инвазивной ИВЛ, смертность составляет от 24 до 53\% [16, 20, 21]. Тяжесть состояния усугубляется частично из-за дистального слизисто-гнойного секрета, препятствующего свободной вентиляции легких. Исследования сыворотки крови и жидкости бронхоальвеолярного лаважа у этих больных выявили высокое содержание НВЛ, которые, в свою очередь, способствуют увеличению вязкости слизисто-гнойной мокроты $[16,20,21]$, что полностью согласуется с полученными нами результатами.

Считается, что чрезмерная воспалительная реакция на SARS-CoV-2 - основная причина тяжести заболевания и смерти у пациентов с COVID-19 - связана с высоким уровнем циркулирующих цитокинов, глубокой лимфопенией и значительной инфильтрацией лейкоцитов в ткани легких, сердца, селезенки, почки и др., что наблюдается при посмертном анализе. Высокий уровень НВЛ, зарегистрированный у этих пациентов, страдающих тяжелой формой ОРДС, рассматривают как критерий риска летального исхода $[17,22,23]$. Полученные нами результаты не противоречат этим данным, но указывают на необходимость дальнейшего детального анализа потенциальных механизмов, ассоциированных с COVID-19.

Представленные данные, несомненно, носят предварительный характер, но в то же время мы можем уверенно заключить, что использование простого и доступного метода, обеспечивающего визуализацию внеклеточных нейтрофильных ловушек, отражающих количество циркулирующих нетозно трансформированных нейтрофилов, их качественный и количественный анализ не только открывает новые возможности для диагностики тяжести состояния пациента, оценки эффективности проводимого лечения, прогноза исхода заболевания, но и способствует выявлению патофизиологической роли нейтрофилов и их внеклеточных структур при COVID-19. В связи с этим перспективны и востребованы дальнейшие исследования, которые позволят осуществить дифференцированный подход к определению групп пациентов, подверженных риску патологического воспаления, разработке инновационных патогенетически обоснованных терапевтических стратегий, возможно, на основе управления цитотоксическими и иммуномодулирующими функциями нейтрофилов.

\section{Заключение}

Результатом проведенной нами работы можно считать выявление некоторых патофизиологических механизмов развития COVID-19, связанных с компартментом нейтрофилов, которые позволили предложить диагностические и прогностические критерии тяжести и исхода заболевания. Установлено, что для больных с коронавирусной инфекцией характерно наличие высокого уровня НВЛ, который в 3 раза и более превышает показатели практически здоровых добровольцев и свидетельствует о сбое иммунных механизмов защиты и развитии неадекватного воспалительного ответа. Повышение нейтрофильных внеклеточных ловушек в мазках цельной крови более 16\% может служить критерием негативного прогноза течения заболевания и риска летального исхода. (๕) 


\section{Дополнительная информация}

\author{
Финансирование \\ Работа проведена без привлечения дополнительного финансирова- \\ ния со стороны третьих лиц.

\section{Конфликт интересов} \\ Авторы декларируют отсутствие явных и потенциальных конфликтов \\ интересов, связанных с публикацией настоящей статьи.

\section{Участие авторов} \\ Д.В. Кассина - сбор и обработка материала, анализ полученных дан- \\ ных, написание текста статьи; И.А. Василенко - концепция и дизайн
}

\begin{abstract}
исследования, анализ и интерпретация результатов исследования написание и редактирование текста статьи, утверждение итогового варианта текста рукописи; А.С. Гурьев - анализ результатов исследования, статистическая обработка данных, редактирование рукописи; А.Ю. Волков - анализ результатов исследования, редактирование рукописи; В.Б. Метелин - формирование групп пациентов, набор клинического материала, анализ и интерпретация результатов исследования. Все авторы внесли существенный вклад в проведение исследования и подготовку статьи, прочли и одобрили финальную версию перед публикацией
\end{abstract}

\section{Литература / References}

1. Li H, Liu Z, Ge J. Scientific research progress of COVID-19/SARS-CoV-2 in the first five months. J Cell Mol Med. 2020;24(12):6558-70. doi: $10.1111 / \mathrm{jcmm} .15364$.

2. Heffernan DS, Evans HL, Huston JM, Claridge JA, Blake DP, May AK, Beilman GS, Barie PS, Kaplan LJ. Surgical Infection Society Guidance for Operative and Peri-Operative Care of Adult Patients Infected by the Severe Acute Respiratory Syndrome Coronavirus-2 (SARS-CoV-2). Surg Infect (Larchmt). 2020;21(4):301-8. doi: 10.1089/sur.2020.101.

3. Leisman DE, Deutschman CS, Legrand M. Facing COVID-19 in the ICU: vascular dysfunction, thrombosis, and dysregulated inflammation. Intensive Care Med. 2020;46(6):1105-8. doi: 10.1007/s00134-020-06059-6.

4. Sun X, Wang T, Cai D, Hu Z, Chen J, Liao H, Zhi L, Wei H, Zhang Z, Qiu Y, Wang J, Wang A. Cytokine storm intervention in the early stages of COVID-19 pneumonia. Cytokine Growth Factor Rev. 2020;53:38-42. doi: 10.1016/j.cytogfr.2020.04.002.

5. Vlachakis PK, Tentolouris A, Tousoulis D, Tentolouris N. Current data on the cardiovascular effects of COVID-19. Hellenic J Cardiol. 2020;61(1):46-8. doi: 10.1016/j. hjc.2020.04.001.

6. Jackson SP, Darbousset R, Schoenwaelder SM. Thromboinflammation: challenges of therapeutically targeting coagulation and other host defense mechanisms. Blood. 2019;133(9): 906-18. doi: 10.1182/blood-2018-11-882993.

7. Twaddell SH, Baines KJ, Grainge C, Gibson PG. The Emerging Role of Neutrophil Extracellular Traps in Respiratory Disease. Chest. 2019;156(4): 774-82. doi: 10.1016/j.chest.2019.06.012.

8. Barnes BJ, Adrover JM, Baxter-Stoltzfus A, Borczuk A, Cools-Lartigue J, Crawford JM, Daßler-Plenker J, Guerci P, Huynh C, Knight JS, Loda M, Looney MR, McAllister F, Rayes R, Renaud S, Rousseau S, Salvatore S, Schwartz RE, Spicer JD, Yost CC, Weber A, Zuo Y, Egeblad M. Targeting potential drivers of COVID-19: Neutrophil extracellular traps. J Exp Med. 2020;217(6):e20200652. doi: 10.1084/ jem.20200652.

9. Masuda S, Nakazawa D, Shida H, Miyoshi A, Kusunoki Y, Tomaru U, Ishizu A. NETosis markers:
Quest for specific, objective, and quantitative markers. Clin Chim Acta. 2016;459:89-93. doi: 10.1016/j.cca.2016.05.029.

10. Gur'ev A, Mosalskaia D, Lopatin A, Volkov A. Prognostic value of cellular markers in sepsis: extracellular DNA traps and platelet count relation. Intensive Care Med Exp. 2019;7 Suppl 3:55. doi: 10.1186/s40635-019-0265-y.

11. Волков АЮ, Мосальская ДВ, Гурьев АС, авторы; ООО «Медтехнопарк», патентообладатель. Способ определения относительного количества этотически трансформированных фагоцитов. Пат. 2712179 Рос. Федерация. Опубл. 24.01.2020. [Volkov AYu, Mosal'skaya DV, Gur'ev AS, authors; OOO «Medtechnopark», assignee. The method of determination of relative number of phagocytes with entotic transformation. Russian Federation patent 2712179. 2020 Jan 24.]

12. Богданова ВД, Андрюков БГ, Ляпун ИН, Бынина МП. Фенотипические субпопуляции нейтрофилов: новые диагностические и иммуномодулирующие стратегии. Здоровье. Медицинская экология. Наука. 2019;(1):5-10. doi: 10.5281/zenodo.2562122. [Bogdanova VD, Andryukov BG, Lyapun IN, Bynina MP. [Phenotype subpopulation of neutrophils: new diagnostic and immunomodulating strategies]. Health. Medical Ecology. Science. 2019;(1):5-10. Russian. doi: 10.5281/zenodo.2562122.

13. Плескова СН, Горшкова ЕН, Боряков $\mathrm{AB}$, Крюков РН. Морфологические особенности быстрого и классического нетоза. Цитология. 2019;61(9):704-12. doi: 10.1134/ S0041377119090098. [Pleskova SN, Gorshkova EN, Boryakov AV, Kriukov RN. [Morphological features of quick and classical netosis]. Tsitologiya [Cytology]. 2019;61(9):704-12. Russian. doi: 10.1134/S0041377119090098.]

14. Papayannopoulos V. Neutrophil extracellular traps in immunity and disease. Nat Rev Immunol. 2018;18(2):134-47. doi: 10.1038/nri.2017.105.

15. Андрюков БГ, Сомова ЛМ, Дробот ЕИ, Матосова ЕВ. Защитные стратегии нейтрофильных гранулоцитов от патогенных бактерий. Здоровье. Медицинская экология. Наука. 2017;(1):4-18. doi: 10.5281/zenodo.345606. [Andryukov BG, Somova LM, Drobot El, Matosova EV. [Defensive strategy of neutrophilic gran- ulocytes against pathogenic bacteria]. Health. Medical Ecology. Science. 2017;(1):4-18. Russian. doi: 10.5281/zenodo.345606.]

16. Zuo Y, Yalavarthi S, Shi H, Gockman K, Zuo M, Madison JA, Blair C, Weber A, Barnes BJ, Egeblad M, Woods RJ, Kanthi Y, Knight JS. Neutrophil extracellular traps in COVID-19. JCI Insight. 2020;5(11):e138999. doi: 10.1172/jci. insight.138999.

17. Tomar B, Anders HJ, Desai J, Mulay SR. Neutrophils and Neutrophil Extracellular Traps Drive Necroinflammation in COVID-19. Cells. 2020;9(6):1383. doi: 10.3390/cells9061383.

18. Yaqinuddin A, Kashir J. Novel therapeutic targets for SARS-CoV-2-induced acute lung injury: Targeting a potential IL-1 $\beta /$ neutrophil extracellular traps feedback loop. Med Hypotheses. 2020;143:109906. Epub ahead of print. doi: 10.1016/j.mehy.2020.109906.

19. Lv D, Xu Y, Cheng H, Ke Y, Zhang X, Ying K. A novel cell-based assay for dynamically detecting neutrophil extracellular traps-induced lung epithelial injuries. Exp Cell Res. 2020;394(2): 112101. Epub ahead of print. doi: 10.1016/j.yexcr.2020.112101.

20. Weber AG, Chau AS, Egeblad M, Barnes BJ, Janowitz T. Nebulized in-line endotracheal dornase alfa and albuterol administered to mechanically ventilated COVID-19 patients: A case series. Preprint. medRxiv. doi: 10.1101/2020.05.13.20087734.

21. Gupta S, Sahni V. The intriguing commonality of NETosis between COVID-19 \& Periodontal disease. Med Hypotheses. 2020;144:109968. Epub ahead of print. doi: 10.1016/j.mehy.2020.109968.

22. Seif S, Ayuna A, Kumar A, Macdonald J. Massive coronary thrombosis caused primary percutaneous coronary intervention to fail in a COVID-19 patient with ST-elevation myocardial infarction. Catheter Cardiovasc Interv. 2020:10.1002/ccd.29050. Epub ahead of print. doi: $10.1002 / c c d .29050$.

23. Thierry AR, Roch B. SARS-CoV2 may evade innate immune response, causing uncontrolled neutrophil extracellular traps formation and multi-organ failure. Clin Sci (Lond). 2020;134(12): 1295-300. doi: 10.1042/CS20200531. 


\title{
Neutrophil extracellular traps: diagnostic and prognostic value in COVID-19
}

\author{
D.V. Kassina' • I.A. Vasilenko',2 • A.S. Gur'ev'1,3 • A.Yu. Volkov³ • \\ V.B. Metelin ${ }^{1,2}$
}

Rationale: An important element of antiviral defense in the pathophysiology of COVID-19 is the innate cell immunity including polymorphonuclear neutrophils prone to netotic transformation. Neutrophils can be not only a marker of acute infection, but, being a source of neutrophil extracellular traps (NET), can play a key role in the development of thrombotic complications leading to acute respiratory insufficiency in COVID-19. Aim: To determine the diagnostic and prognostic value of NET levels in patients with COVID-19. Materials and methods: We monitored NET levels in peripheral blood of 34 patients with COVID-19 (mean age, $67 \pm 15.8$ years), admitted to MONIKI hospital. The control group consisted of 54 healthy volunteers (mean age, $52 \pm 11.5$ years). Whole blood samples of $2 \mu \mathrm{L}$ each were used for the preparation of monolayer smears (Giemsa stain) and calculation of at least 200 cell structures including native intact and transformed neutrophils (MECOS-C2 microscope, Medical computer systems). Results: Patients with COVID-19 had higher NET levels, compared to those in healthy controls: $14.5 \%(2.9-28.6 \%)$ vs. $5.0 \%(1.8-11.9 \%, p<0.0001)$. The patients who were on non-invasive respiratory support (23.5\%) had a NET level of $12 \%$ (8.1$22.3 \%$ ), whereas those on invasive mechanical ventilation $(17.6 \%)$ had a 1.5 -fold higher NET level of $17.9 \%(12.3-28.2 \%)(p<0.05)$. In the patients who died $(11.8 \%$ of the cases), the NET level amounted to $19 \%(16.5-26 \%, p<0.05)$. Monitoring of blood
NET levels was performed in 9 patients from the day of admittance to the day of their transfer to the intensive care unit/discharge/death. It was shown that a decrease of NET levels mirrors an improvement of the patient's clinical condition and efficacy of his/hers treatment. On the opposite, an increase of NET levels can indicate a deterioration and risk of unfavorable course. Conclusion: We have identified some pathophysiological mechanisms in COVID-19, related to the neutrophil compartment. Patients with coronavirus infection are characterized by high NET levels which is at least 3 -fold higher than that in healthy volunteers. This indicates an abnormality in immune host defense and development of an inadequate inflammatory response. An increase of NET in whole blood smears of more than $16 \%$ can be a criterion of an unfavorable prognosis of the disease course and the risk of death.

Key words: COVID-19, neutrophil extracellular traps, NETosis

For citation: Kassina DV, Vasilenko IA, Gur'ev AS, Volkov AYu, Metelin VB. Neutrophil extracellular traps: diagnostic and prognostic value in COVID-19. Almanac of Clinical Medicine. 2020;48(S1):S43-50. doi: 10.18786/2072-0505-2020-48-029.

Received 13 July 2020; revised 28 July 2020; accepted 29 July 2020; published online 11 August 2020

\section{Conflict of interests}

The authors declare no obvious and potential conflicts of interests related to the publication.

\section{Authors' contributions}

D.V. Kassina, data collection, management and analysis, text writing; I.A. Vasilenko, the study concept and design, analysis and interpretation of the study results, text writing and editing, approval of the final version of the manuscript; A.S. Gur'ev, analysis of the study results, statistical analysis, text editing; A.Yu. Volkov, analysis of the study results, text editing; V.B. Metelin, recruitment of the patient groups, clinical data collection, analysis and interpretation of the study results. All the authors have made their significant contributions to the research and preparation of the article, have read and approved the final version before submission.

Darya V. Kassina - Research Fellow, Scientific and Research Laboratory'; ORCID: https://orcid.org/0000 0002-6759-9121

Irina A.Vasilenko - MD, PhD, Professor, Leading Research Fellow, Scientific and Research Laboratory' Professor, Chair of Applied Mathematics and Programming ${ }^{2}$; ORCID: http://orcid.org/0000-00026374-9786

$\triangle$ 33/1 Sadovnicheskaya ul., Moscow, 115035 , Russian Federation. Tel.: +7 (495) 9515497. E-mail: vasilenko0604@gmail.com

Alexander S. Gur'ev - PhD, Senior Research Fellow, Scientific and Research Laboratory'; Research Fellow33 ${ }^{3}$ ORCID: https://orcid.org/0000-0001-88237819

Alexey Yu. Volkov - PhD (in Phys. and Math.), General Director ${ }^{3}$; ORCID: https://orcid.org/0000 0001-5110-553X

Vladislav B. Metelin - PhD (in Biol.), Leading Research Fellow, Scientific and Research Laboratory'; Associate Professor, Chair of Philosophy, Manusology and Theology ${ }^{2}$; ORCID: http://orcid.org/0000-00030600-5729

Moscow Regional Research and Clinical Institute (MONIKI); 61/2 Shchepkina ul., Moscow, 129110, Russian Federation

${ }^{2}$ The Kosygin State University of Russia;

33/1 Sadovnicheskaya ul., Moscow, 115035, Russian Federation

${ }^{3}$ Medtechnopark; 8-2-383 Profsoyuznaya ul., Moscow, 117292, Russian Federation 